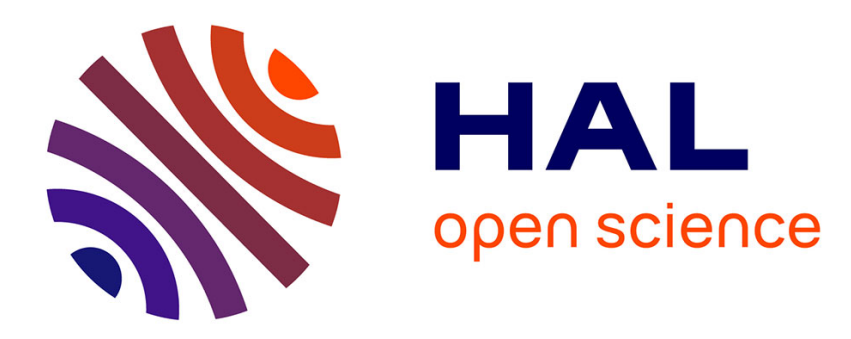

\title{
L'organisation politique des Kwagul méridionaux au XIXe siècle
}

\author{
Marie Mauzé
}

\section{To cite this version:}

Marie Mauzé. L'organisation politique des Kwagul méridionaux au XIXe siècle. Journal de la Société des américanistes, 1989, 75, pp.173-192. halshs-00714453

\section{HAL Id: halshs-00714453 \\ https://shs.hal.science/halshs-00714453}

Submitted on 4 Jul 2012

HAL is a multi-disciplinary open access archive for the deposit and dissemination of scientific research documents, whether they are published or not. The documents may come from teaching and research institutions in France or abroad, or from public or private research centers.
L'archive ouverte pluridisciplinaire HAL, est destinée au dépôt et à la diffusion de documents scientifiques de niveau recherche, publiés ou non, émanant des établissements d'enseignement et de recherche français ou étrangers, des laboratoires publics ou privés. 


\section{L'organisation politique des Kwagul méridionaux au XIXe siècle} In: Journal de la Société des Américanistes. Tome 75, 1989. pp. 173-192.

Citer ce document / Cite this document :

Mauzé Marie. L'organisation politique des Kwagul méridionaux au XIXe siècle. In: Journal de la Société des Américanistes. Tome 75, 1989. pp. 173-192.

doi : $10.3406 /$ jsa. 1989.1348

http://www.persee.fr/web/revues/home/prescript/article/jsa_0037-9174_1989_num_75_1_1348 


\section{Abstract}

The political organization of the Southern Kwagul in the 19th century. The author attempts to analyse the process of transformations undergone by the Kwagul political system during a period extending from contact (end of the 18th century) to the end of 19th century. The traditional organization in numaym, the basic social units, is in the process of being replaced by a double system which on the one hand, maintains the numaym' $s$ identity while on the other hand integrates them into larger tribal units. The latter are included in larger ceremonial congregations. Whereas, within the tribe, social cohesion is centered on the numaym, defense is assured by regrouping the numaym. Within the context of warfare, the tribe functions as a political unit. Secret societies contribute to the balance of power between the numaym and the tribe. The notion of « chieftainship » applies only partially to these political units.

\section{Resumen}

La organization política de los Kwagul méridionales en el siglo XIX.El autor ha intentado captar la dinámica de las transformaciones que han afectado el sistema politico de los Kwagul (costa noroeste) desde la época del contacto (fines de siglo xviii) hasta fines del siglo xix. La organizaciôn tradicional en numaym, unidades sociales de base, ha sido suplantada progresivamente por un doble sistema. Este conserva por un lado la identitad del numaym y por otro lo intégra a unidades mayores, las tribus, que forman a su vez grandes conjuntos ceremoniales. Al interior de la tribu, el centro de cohesion social es siempre el numaym y el agrupamiento de estas unidades obedece, entre otras causas, a exigencias defensivas ; en el contexto de la guerra la tribu constituye la unidad política. Las sociedades sécrétas contribuyen a mantener el equilibrio del poder entre numaym y tribu, unidades politicas a las que no conviene el término jefatura.

\section{Résumé}

On s'efforce de saisir la dynamique des transformations subies par le système politique des Kwagul (côte nord-ouest) depuis le contact (fin du xvin* siècle) jusqu'à la période coloniale (fin xixe siècle). L'organisation initiale en numaym, unités sociales de base de type " maison », est progressivement supplantée par un double système, qui d'une part, maintient l'intégrité des numaym, et, d'autre part, les intègre dans des unités plus larges, les tribus, qui vont elles-mêmes se regrouper dans de larges ensembles cérémoniels. Si, au sein de la tribu, le lieu de cohésion sociale demeure le numaym, le regroupement des numaym en tribus, répond, entre autres, à des impératifs de défense : par le rôle qu'elle joue dans les guerres, la tribu prend figure d'unité politique. Les sociétés secrètes interviennent comme des facteurs d'équilibre entre le numaym et la tribu : à chacune de ces deux unités politiques, le terme de « chefferie » ne s'applique qu'imparfaitement.

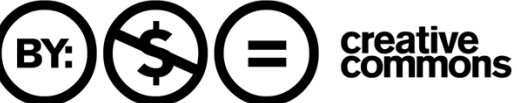




\title{
L'ORGANISATION POLITIQUE DES KWAGUL MÉRIDIONAUX AU XIX ${ }^{e}$ SIÈCLE
}

\author{
Marie MAUZÉ*
}

\begin{abstract}
On s'efforce de saisir la dynamique des transformations subies par le système politique des Kwagul (côte nord-ouest) depuis le contact (fin du XviII ${ }^{e}$ siècle) jusqu'à la période coloniale (fin $\mathrm{XIX}^{e}$ siècle). L'organisation initiale en numaym, unités sociales de base de type « maison ", est progressivement supplantée par un double système, qui d'une part, maintient l'intégrité des numaym, et, d'autre part, les intègre dans des unités plus larges, les tribus, qui vont elles-mêmes se regrouper dans de larges ensembles cérémoniels. Si, au sein de la tribu, le lieu de cohésion sociale demeure le numaym, le regroupement des numaym en tribus, répond, entre autres, à des impératifs de défense : par le rôle qu'elle joue dans les guerres, la tribu prend figure d'unité politique. Les sociétés secrètes interviennent comme des facteurs d'équilibre entre le numaym et la tribu : à chacune de ces deux unités politiques, le terme de « chefferie » ne s'applique qu'imparfaitement.
\end{abstract}

\section{La organización politica de los Kwagul meridionales en el siglo XIX.}

El autor ha intentado captar la dinámica de las transformaciones que han afectado el sistema político de los Kwagul (costa noroeste) desde la época del contacto (fines de siglo XVIII) hasta fines del siglo XIX. La organización tradicional en numaym, unidades sociales de base, ha sido suplantada progresivamente por un doble sistema. Este conserva por un lado la identitad del numaym y por otro lo integra a unidades mayores, las tribus, que forman a su vez grandes conjuntos ceremoniales. Al interior de la tribu, el centro de cohesión social es siempre el numaym y el agrupamiento de estas unidades obedece, entre otras causas, a exigencias defensivas; en el contexto de la guerra la tribu constituye la unidad política. Las sociedades secretas contribuyen a mantener el equilibrio del poder entre numaym y tribu, unidades políticas a las que no conviene el término jefatura.

The political organization of the Southern Kwagul in the 19th century.

The author attempts to analyse the process of transformations undergone by the Kwagul political system during a period extending from contact (end of the 18 th century) to the end of 19 th century. The traditional organization in numaym, the basic social units, is in the process of being replaced by a double system which on the one hand, maintains the numaym's identity while on the other hand integrates them into larger tribal units. The latter are included in larger ceremonial congregations. Whereas, within the tribe, social cohesion is

* Laboratoire d'anthropologie sociale, Paris.

J.S.A. 1989, LXXV : p. 173 à 192. 
centered on the numaym, defense is assured by regrouping the numaym. Within the context of warfare, the tribe functions as a political unit. Secret societies contribute to the balance of power between the numaym and the tribe. The notion of " chieftainship " applies only partially to these political units.

Dans Des Clans aux Empires (1922), écrit en collaboration avec A. Moret, Geoges Davy a analysé avec justesse (chapitre vi) « les conditions de l'individualisation du pouvoir " chez les Kwagul méridionaux (Kwakiutl). En rendant compte du passage d'une organisation de type "totémique » à une autre forme d'organisation correspondant pour lui à une situation d'émergence de la royauté, Davy met en évidence des aspects saillants de l'organisation sociale et politique des Kwagul. Il est intéressant de montrer en quoi l'analyse de Davy touchant aux populations de la côte nord-ouest du Pacifique, superbement ignorée par les auteurs nord-américains, mérite plus d'attention que celle qui lui a été prêtée jusqu'ici, même si le vocabulaire de l'auteur nous semble aujourd'hui quelque peu dépassé.

En s'appuyant essentiellement sur les travaux de Boas, Davy montre que dans les sociétés de la côte nord-ouest et plus particulièrement chez les Kwagul, le pouvoir a fait l'objet d'une progressive concentration et individualisation, sur fond de « masculinisation » de la filiation, à travers la détention et la transmission de biens symboliques, de telle sorte que le «chef » en est venu à être à la fois l' " ancêtre » du groupe et l'héritier des ancêtres. Les biens symboliques - les blasons, par exemple, que Davy appelle des « totems »-, changent de nature en devenant des titres qui sont transmis ${ }^{1}$. La détention héréditaire du pouvoir s'accompagne de l'établissement d'un lien contractuel associant le chef et ses gens, qui s'exprime dans le potlatch ${ }^{2}$. C'est dans le potlatch qu'est mise en jeu la légitimité acquise contre la légitimité héritée. Le potlatch, écrit Davy, « ouvre les voies par où s'introduira l'individu sur la scène sociale, puisqu'il brise les cadres héréditaires et rigides au sein desquels nulle place n'était faite à la concurrence ni à l'adaptation individuelle, et qu'il érige la rivalité en institution sociale ") (1922 : 109) ${ }^{3}$. C'est dans le potlatch que le pouvoir met en œuvre les principes de sa légitimation et de sa reproduction : individualisation et accentuation du prestige de la fonction de chef, réactualisation de la hiérarchie sociale, manifestation du pouvoir tributaire du chef et réaffirmation du contrat liant le chef et son groupe, etc. L'un des aspects de la détention du pouvoir chez les $\mathrm{K}$ wagul réside dans la capacité et la propension qu'elle recèle à contrôler et à manipuler des alliances, qui sont jouées contre la filiation. "Le mariage est (...) le substitut de l'hérédité » (Davy, ibid. : 105). Soulevé par Davy, ce problème été plus amplement développé par C. Lévi-Strauss dans son article sur "L'organisation sociale des Kwakiutl » (1979), dans lequel est traitée la problématique de la « maison ».

Le processus d'individualisation du pouvoir au sein de ce que Davy appelle le clan - né de la substitution du clan local au clan totémique (1922: 70) — se réalise pleinement dans le cadre des confréries religieuses qui, écrit-il, " semblent être pour la souveraineté un foyer privilégié d'individualisation " (ibid. : 118) en raison de leur organisation hiérarchisée. Sans pour autant suivre pas à pas Davy dans son analyse, citons une phrase clé de sa démonstration : 
« Et ce cadre (la confrérie) quasi-féodal est précisément destiné à contenir la hiérarchie et les relations de genre vassalitique qui conviennent à ces sociétés oligarchiques dominées par des chefs où le pouvoir apparaît comme concentré et individualisé et tout prêt pour cette unification finale : la royauté " (ibid. : 103).

Peuple de pêcheurs-chasseurs-cueilleurs, pratiquant le stockage des aliments (Testart 1982), les Kwagul méridionaux, au $\mathrm{xIX}^{\mathrm{e}}$ siècle, sont répartis en une vingtaine de tribus, correspondant chacune à un groupement local ou village d'hiver. Ces tribus sont divisées en unités sociales plus petites, les numaym, généralement considérées comme les unités fondamentales de l'organisation sociale et politique (Boas 1920, 1966; Drucker 1983; Lévi-Strauss 1979). Le système à numaym est celui qui prévaut pendant la période profane d'été (avril-octobre), vouée aux activités de subsistance, les activités cérémonielles d'hiver (novembremars) étant placées sous le contrôle des sociétés secrètes ou sociétés de danse. Tribu, numaym et société secrète sont organisés selon une hiérarchie de rangs. Ces hiérarchies relèvent d'un modèle unique, mais sont fondées sur des principes distincts, comme nous le verrons plus loin.

\section{LE NUMAYM}

Faute de trouver le terme ad hoc dans le vocabulaire anthropologique pour désigner l'unité de base de l'organisation sociale des Kwagul, Boas (1920:115) choisit d'utiliser son nom indigène, qui signifie « ceux de la même sorte " (1966 : $37)^{4}$. Le numaym est un groupe de parenté dont l'ossature est censée être constituée par un groupe de descendance patrilinéaire issu d'un ancêtre mythique commun, mais au sein duquel les faits d'appartenance et de transmission montrent qu'y prévaut une indifférenciation de la filiation. Lévi-Strauss, en empruntant au Moyen-Age européen le mot « maison » et en l'utilisant pour rendre compte de ce qu'est le numaym, a placé l'organisation sociale kwagul sous un éclairage nouveau. Pour Lévi-Strauss, le numaym est :

«... une personne morale détentrice d'un domaine composé à la fois de biens matériels et immatériels, qui se perpétue par la transmission de son nom, de sa fortune et de ses titres en ligne réelle ou fictive, tenue pour légitime à la seule condition que cette continuité puisse s'exprimer dans le langage de la parenté et de l'alliance, et, le plus souvent, des deux ensemble » (1979: 177).

Ainsi, le numaym est un groupe pour lequel « la lignée noble ne coïncide pas avec la lignée agnatique et (...) est même souvent dépourvue de base biologique " (ibid.). Le numaym peut certes être défini comme un groupe de descendance, mais ce qui marque la particularité de chaque numaym, son identité, c'est un certain nombre de positions, chacune étant associée à un nom, à un rang et aux privilèges qui leur sont attachés; nom et rang ont une existence indépendante des individus auxquels ils sont temporairement liés (Boas 1966 : 50).

Le numaym comprend des nobles, des gens du commun ${ }^{5}$ - ceux qui ne détiennent pas des titres (Boas, 1899, 1920, 1966) - et des esclaves ${ }^{6}$. La différence 
entre le statut de noble et celui d'homme du commun peut être mise en évidence dans le contexte du potlatch, d'une part, et dans celui de l'activité symbolicorituelle, d'autre part. Au sein du numaym, nobles et gens du commun sont soumis à des prélèvements d'importance comparable opérés sur leurs biens de subsistance par le chef, en vue de la préparation des potlatch : à cet égard, nobles et gens du commun sont assujettis aux mêmes exigences. La différence se manifeste lors de distributions, car alors seuls les nobles, après le chef, reçoivent des biens; les gens ordinaires ne reçoivent rien. La différence entre nobles et gens du commun est ici relative : les uns et les autres donnent (à leur chef de numaym), mais seuls les premiers reçoivent (des chefs des autres numaym). En matière rituelle, la différence est absolue : seuls les nobles contrôlent l'usage du patrimoine symbolique (noms, blasons, chants, légendes, danses, etc.). Formellement, eux seuls, en outre, du fait de leur rang de naissance et de ce dont ils sont dépositaires, ont vocation à occuper les positions hiérarchiques du numaym. Le numaym est une unité sociale à rangs caractérisée par l'existence d'une hiérarchie entre les individus, marquée par une série continue et graduée de statuts telle que deux statuts ne sont jamais équivalents entre eux. Chaque statut est en quelque sorte à lui-même sa propre catégorie (Terray $1985: 110$ ). La hiérarchie s'exerce à l'intérieur d'un tissu social homogène, celui de la parenté. Un noble (pl. nax̌sala), ou " real one ", ne transmet pas nécessairement son statut à l'ensemble de sa progéniture : ainsi, aucun nom n'est transmis au delà du cinquième enfant (cf. Boas $1920: 117)^{7}$. Les modalités de transmission des titres au sein de la noblesse kwagul sont plus proches - si l'on permet la comparaison - de celles qui prévalent dans la noblesse anglaise que de celles qui sont propres à la noblesse française. En France, l'annoblissement d'un individu vaut pour lui-même, sa famille et ses descendants : l'appartenance à la noblesse est une marque collective. En Angleterre, au contraire, l'état de noblesse d'un individu est transmis au conjoint, et, dans la descendance, il ne va qu'au fils aîné (Carroll $1980: 211-212$ ). Chez les Kwagul, le droit au titre et/ou à la fonction prévaut sur le lien de sang. La noblesse kwagul est principalement une noblesse de titulature : c'est le nom de la position que l'on occupe au sein du numaym qui confèrent la qualité de noble. On pourrait presque dire que le " degré de noblesse " qui caractérise un individu est en rapport direct avec la qualité ou la valeur intrinsèque du nom qu'il détient au sein du numaym (Mauzé 1988). On est donc "plus ou moins" noble selon sa place dans la hiérarchie; au plus haut de la hiérarchie du numaym, le " noble " par excellence est exemplairement le chef. Le prestige d'un individu se mesure à l'aune de la somme totale des privilèges qu'il détient non seulement au sein du numaym mais aussi dans la hiérarchie des sociétés secrètes. Il y a, dans une certaine mesure, homologie entre les hiérarchies des numaym et des sociétés secrètes et correspondance, d'un numaym à une société, entre les positions que peut occuper un même individu; les statuts sont cumulatifs et c'est l'accumulation de statuts qui est génératrice de prestige. À ce propos, Sapir (1915 : 114) souligne : "le rang n'est pas un statut permanent; il résulte d'une situation acquise par l'héritage de privilèges multiples, théoriquement indépendants, dans la plupart des cas cumulatifs, qui peuvent se transmettre séparément de génération en génération ".

Comme nous l'avons dit, des noms - en nombre limité - attachés au numaym constituent son identité. Ces noms forment une partie du patrimoine symbolique 
inaliénable du numaym : ils sont et ne peuvent cesser d'être sa propriété (Boas \& Hunt 1921 : 823-824). Ces noms sont transmis au sein de la lignée noble, de père en fils, ou, plus exceptionnellement, de père en fille. Des noms constituant un autre ensemble peuvent circuler, par le mariage, de beau-père à gendre, par l'intermédiaire de la fille, pour être transmis aux premiers enfants du couple. Font partie de ce second ensemble des noms appartenant aux confréries religieuses (cf. ci-dessous). L'acquisition de tels noms, au cours du XIX ${ }^{e}$ siècle, a été l'objet d'enjeux politiques importants : les alliances matrimoniales étaient conclues dans le but d'obtenir le droit à être initié à une danse particulière et à bénéficier des privilèges liés à cette initiation. La détention de noms appartenant aux sociétés secrètes était un élément esentiel du statut personnel.

Ce qui est en jeu dans la succession, c'est la continuité du numaym, qui se reproduit et puise sa légitimité dans la manipulation de la filiation et de l'alliance. Que l'alliance entre deux ou plusieurs lignées soit durable ou non, ce qui compte, c'est la préservation ou mieux encore l'agrandissement du patrimoine de la « maison ». À ce propos, Lévi-Strauss (1979: 188-189) remarque :

« Descendance patrilinéaire et descendance matrilinéaire, filiation et résidence, hypergamie et hypogamie, mariage proche et mariage lointain, race et élection : toutes ces notions, qui servent d'habitude aux ethnologues pour distinguer les uns des autres les divers types connus de société, se rassemblent dans la maison, comme si l'esprit (au sens du Xvill siècle) de cette institution traduisait, en dernière analyse, un effort pour transcender, dans tous les domaines de la vie collective, des principes théoriquement inconciliables ».

Quels que soient les principes mis en œuvre, c'est le patrimoine qui doit rester intact : comme le note justement Sapir (1915: 118), qui se fait l'interprète d'un numaym, «les gens vont et viennent, je demeure ". Dans ces «maisons ", correspondant à des familles nobles, la transmission des privilèges par l'une des deux lignées ou par les deux à la fois prime sur tout autre règle pour assurer la reproduction du groupe, le maintien de son patrimoine, la conservation de son identité. À la fin du $\mathrm{XIX}^{\mathrm{e}}$ siècle, certains individus nobles parviendront à occuper des positions dans plusieurs numaym au sein d'une même tribu ou même dans plusieurs tribus, en exploitant toutes les possiblités de la filiation et de l'alliance.

Le numaym est une unité territoriale. Chaque numaym dispose d'un territoire ${ }^{8}$ bien délimité qui correspond à un ensemble de lieux, reliés entre eux par un réseau d'itinéraires maritimes, comprenant un village, des sites de pêche (tronçon de rivière, plage), des lieux de cueillette, des terrains de chasse, sur lesquels les droits d'exploitation sont jalousement protégés (Boas 1966: 35). Chaque numaym revendique comme lieu d'origine une localité déterminée (Boas 1920 : 111; 1966 : 42), à laquelle correspondent les privilèges obtenus par l'ancêtre fondateur du groupe : les privilèges sont attachés à la terre (Sapir 1915:117). Le droit exclusif à disposer d'un territoire, d'une terre, est ainsi un élément essentiel à l'identité du groupe.

Le numaym est une unité de production et de consommation. Les ressources du territoire sont la propriété du numaym en son entier. C'est le chef, en tant que gestionnaire du patrimoine foncier, qui contrôle l'accès aux ressources. Si les nobles et les gens du commun exploitent de manière équivalente les sites de production, le chef prélève une part du produit du travail de chaque maisonnée, ce 
qui lui permet de constituer un surplus que lui seul détient. Les biens alimentaires ainsi stockés sont destinés à être distribués au cours de fêtes ou de potlatch. Les données ethnographiques dont nous disposons semblent indiquer que lors de la période de constitution des stocks alimentaires, les prélèvement s'effectuaient de manière " égalitaire " sur les nobles et les gens du commun, en fonction de quotas déterminés (Boas 1921 : 1333-1338). En revanche, lorsqu'il s'agissait d'objets de prestige ou lorsque le prélèvement était différé - lorsqu'il intervenait immédiatement avant l'organisation d'un potlatch -, chacun donnait à proportion de l'importance de son statut : dans ce cas, ce sont les nobles qui cédaient de nouveaux biens au chef (Mauzé 1986 : 29). Le chef kwagul a ainsi la capacité "politique " de mobiliser des biens du numaym : une fois qu'il les a réunis, il a l'autorité de manipuler ces biens à son profit de manière à en tirer le plus grand prestige. Il lui arrive de faire preuve de prodigalité à l'égard de ses gens en leur offrant à tous de la nourriture, au cours des fêtes. En cas de famine, le chef a l'obligation de nourrir les membres de son groupe en puisant dans ses stocks. $\mathrm{Ce}$ rôle de détenteur d'une réserve alimentaire n'est rempli par le chef qu'en des occasions exceptionnelles; il est impropre de parler de redistribution dans le contexte socio-économique kwagul. En effet, le système d'accumulation et de distribution, générateur de réciprocité, est orienté vers les groupes voisins, partenaires dans le potlatch : ce n'est pas un système régulateur d'auto-subsistance contrôlé par un pouvoir. Le chef accumule, donne et reçoit au nom de son groupe, mais en réalité il agit en son nom propre. Les biens du chef invitant vont aux chefs invités et aux personnages de haut rang: les gens du commun ne reçoivent rien (Mauzé 1986 : 29-30; Testart 1982 :207-209). Tout comme la hiérarchie, le système d'exploitation opère au sein de la parenté : c'est la validation d'un statut à l'extérieur qui permet au nom de la solidarité intra-numaym de légitimer l'exploitation à l'intérieur (Lévi-Strauss $1984: 148$; Testart 1985: 245-246). La richesse du chef apparaît comme devant être un élément essentiel de la " chefferie " (chieftainship) kwagul. Si le pouvoir peut être défini en termes de prestige, le prestige est indissociable du rang et de la richesse.

Toutefois, si la description précédente relève d'une situation qui avait cours encore pendant la première partie du $\mathbf{X I X}^{\mathrm{e}}$ siècle, les choses changent dès les années 1850. En effet, le numaym, en tant qu'unité de production, perd de son importance en raison de la possibilité d'accès à de nouvelles ressources, grâce à la traite des fourrures dans un premier temps et par la suite, vers 1870, grâce au travail salarié. L'obtention de biens de prestige passe par un circuit qui n'est plus contrôlé directement par le chef de numaym; en outre, elle n'est plus associée à l'exploitation exclusive de son territoire par un groupe. Le mode d'accès à la richesse s'individualise, la solidarité du groupe est mise à mal. L'accumulation de biens en vue d'une distribution lors d'un potlatch est devenue une affaire individuelle, de telle sorte que dans les années 1880 , lorsqu'un effort matériel est demandé à l'ensemble du groupe comme cela se faisait autrefois, les membres du numaym n'hésitent pas à parler d' "impôt " (Lando $1988: 132)^{9}$. 


\section{LA TRIBU}

Les analyses du « politique » chez les Kwagul méridionaux mettent généralement l'accent sur la place qu'occupe le numaym dans l'organisation socio-politique. Le numaym, unité territoriale, économique, etc., est considéré comme étant autonome aussi bien en temps de paix qu'en temps de guerre (Drucker 1983 : 88). Ceci étant posé, nous devons considérer une autre unité socio-politique, plus large que le numaym, qui est la tribu.

Au cours du XIX ${ }^{e}$ siècle, la tribu s'affirme comme étant une unité à la fois militaire (Curtis 1915) et cérémonielle (Drucker et Heizer 1967; Drucker 1983). En vérité, les auteurs qui ont étudié l'organisation politique des Kwagul n'ont généralement pas très bien su quoi faire de la tribu : on s'est souvent contenté de la considérer comme une fédération de numaym indépendants les uns des autres, ne disposant donc que d'une faible capacité d'intégration des éléments qui la constituent.

Il nous faut opérer ici un retour à l'histoire. À une époque qu'il est difficile de dater mais qui est vraisemblablement antérieure aux années 1820-1830, c'est-à-dire quelques décennies après le contact (1774) (Tolmie 1963 : 317-319) ${ }^{10}$, des formations plus larges que le numaym, que la tradition anthropologique baptisera « tribus ", font leur apparition. Boas (1966: 41) définit les tribus ${ }^{11}$ commé étant « des communautés résidant chacune dans un village d'hiver et qui s'associent à l'occasion des guerres et des rituels. Ce sont des communautés villageoises qui portent souvent le nom du lieu qu'elles habitent ". Selon Boas (1890:69; 1966 : 46), le regroupement de numaym en villages, lors de la période « historique » (postérieure au contact), a été probablement dicté par le souci qu'éprouvaient des groupes affaiblis par la guerre et les maladies de se donner une assise nouvelle : on a là le résultat d'une évolution récente. Dès la fin du Xvir siècle, en effet, les populations de la côte nord-ouest sont touchées par des épidémies de variole (Boyd 1985), comme en témoignent les récits des premiers voyageurs et traitants de fourrures; l'épidémie la plus catastrophique fut celle de 1862 qui, selon Boyd (ibid. : 262), tua $69 \%$ de la population kwagul (cf. aussi Codere 1950). L'introduction des armes à feu et l'intensification des guerres pour le contrôle du commerce des fourrures ${ }^{12}$, entre la fin du $\mathrm{XVIII}^{\mathrm{e}}$ siècle et les années 1860 , date du début de la période coloniale, contribuèrent au déclin de la population : certains groupes furent complètement anéantis (Boas 1966 : 47). Dans ce contexte de décroissance démographique, des numaym voisins pratiquant ensemble l'échange de femmes et de biens se regroupent afin d'assurer leur sécurité et finalement leur survie (Dawson 1887 : 66; Donald \& Mitchell 1975). La tribu kwagul est formée d'unités agrégées les unes aux autres, sans pour autant que cette association leur fasse perdre leur individualité, et semble-t-il, une large part de leur autonomie. La tribu, qui réunit des numaym pendant plusieurs mois de l'année, est, d'abord, une unité résidentielle : elle correspond à une communauté villageoise. Le fait tribal est basé sur la recherche d'un équilibre entre numaym. Chaque tribu, a son tour, est en relation d'opposition ou de complémentarité avec d'autres unités sociales de même nature, d'autres tribus, dans les fêtes, les potlatch et la guerre. 
Avant de traiter des relations entre tribus, il faut examiner quelle était la nature des relations existant entre les numaym à l'intérieur d'une même tribu. Plusieurs faits peuvent être mis en évidence : 1) La tribu intègre en son sein un nombre limité de numaym, généralement quatre ou cinq, sept au plus (Boas 1897 : 329-332; 1966 : 38-41). 2) Les numaym ont des territoires géographiquement voisins, situés dans un environnement écologique homogène, ce qui interdit toute prédominance économique d'un groupe sur l'autre : il $\mathrm{y}$ a accès au même type et à la même quantité de ressources alimentaires; pourrait-on peut-être déceler des disparités économiques entre numaym en fonction de la situation des sites de pêche selon, notamment, qu'ils sont situés à l'amont ou à l'aval d'une rivière ou d'un inlet ? 3) Les numaym associés ne sont pas nécessairement liés entre eux généalogiquement (Boas $1966: 44$ ); il existe cependant quelques cas de paires de numaym issues d'un même ancêtre, ou ancêtres frères (Boas 1905 : 179). 4) Il apparait que les numaym d'une tribu ont généralement entretenu entre eux, au cours de leur histoire, des relations matrimoniales et économiques permanentes et privilégiées. 5) Les associations entre numaym sont relativement stables, mais il arrive cependant qu'un numaym se sépare d'une tribu pour s'agréger à une autre. 6) Alors que le numaym hiérarchise des individus, la tribu hiérarchise des numaym. Cette question de la hiérarchisation des numaym se pose notamment à propos de la problématique d'ensemble de l'individualisation du pouvoir. La hiérarchie des numaym à l'intérieur de la tribu est fondée, dans l'histoire, sur l'ordre chronologique d'affiliation à la tribu; au terme du processus de formation de la tribu, la hiérarchie des numaym tend à être légitimée par le mythe (Boas $1897: 339)^{13}$. 7) En théorie, l'ordre hiérarchique des numaym à l'intérieur de la tribu est immuable, mais il peut cependant être remis en question (Curtis $1915: 141$ ); le rang d'un numaym se manifeste, dans l'étiquette cérémonielle, par l'ordre des préséances dans la distribution des biens dans les fêtes et potlatch ${ }^{14}$ ). 8) Le chef de tribu est le chef du numaym le plus élevé; sa position n'est généralement pas révocable. 9) Le territoire de la tribu ne correspond pas à la somme des espaces des numaym : selon Boas, cette conception totalisante d'un " territoire tribal " est étrangère aux Indiens. Il semble qu'on ne puisse parler de territorialité tribale qu'en référence au village d'hiver, lieu fixe commun à tous les numaym de la tribu, bien que chaque numaym ait disposé d'un " quartier " qui lui était propre. 10) Il n'y a pas d'économie globale de la tribu : le chef de tribu n'exerce aucun contrôle sur la production de l'ensemble des numaym; cependant, au nom de la solidarité interne à la tribu, dans une contexte de rivalité avec une autre tribu, le chef peut solliciter une certaine forme de coopération de la part des chefs et des nobles de chacun des numaym. 11) Le chef de tribu ne dispose d'aucun moyen direct de pression sur les chefs de numaym pour faire prévaloir son autorité. Le chef de tribu semble donc n'avoir pas disposé d'un pouvoir politique à strictement parler, ce qui ne l'empêchait pas d'être, par définition, la figure centrale de la tribu : les relations entre numaym s'organisaient autour de lui à chaque fois que le destin global de la tribu - ou la somme des intérêts des numaym - était en cause. Le chef était un médiateur et un garant et donc était bien finalement un "chef ". Le cas de la guerre est évocateur à cet égard. Le chef ne déclarait pas la guerre au nom de la tribu : les guerres naissaient le plus souvent à l'initiative d'un numaym. Mais Boas $(1897,1966)$ et Curtis $(1915: 98-124)$ notent qu'un numaym ne se lançait jamais 
seul dans une guerre, qu'il bénéficiait toujours de l'aide des autres numaym de sa tribu; à côté des guerriers professionnels, les guerriers occasionnels comprenaient des représentants du numaym qui était au départ de la guerre et des autres numaym. Sans doute ne peut-on en pareil cas parler de guerre « tribale ", en ce que la tribu en serait l'acteur unique, mais il demeure que, dans la guerre, la solidarité entre numaym entrainait dans tout conflit la tribu tout entière, sous l'autorité morale de son chef. De fait, la guerre est ainsi l'affaire de l'ensemble de la tribu ${ }^{15}$; qu'elle soit offensive ou défensive, elle est même un facteur important de cohésion de la tribu (Donald et Mitchell 1975 : 342). En conclusion, si le numaym demeure, au sein de la tribu, le cadre privilégié de l'activité économique, matrimoniale et sociale, la tribu, malgré son hétérogénéité, inhérente aux conditions habituelles de sa formation, apparait comme une unité résidentielle, cérémonielle et - on a vu dans quelles conditions - militaire, le chef de tribu étant le personnage emblématique de l'intégration tribale.

$\mathrm{Au}$ cours du $\mathrm{XIX}^{\mathrm{e}}$ siècle, les $\mathrm{Kwagul}$ se répartissent progressivement entre six grandes unités cérémonielles (Drucker et Heizer 1967; Goldman 1975) qui sont des regroupements de tribus spatialement proches, entretenant entre elles des relations matrimoniales privilégiées. Au départ, les potlatch inter-tribaux n'associent pas ensemble toutes les tribus d'une même unité; celles-ci se regroupent par paires inégalitaires, les tribus ainsi appariées étant, selon les circonstances, en relation de rivalité l'une par rapport à l'autre, ou, ensemble, en relation de solidarité par rapport à d'autres paires de la même grande unité cérémonielle. Ici encore, comme pour la guerre (cf. ci-dessus), il faut aller du numaym vers la tribu. Le référent des diverses formes de potlatch, c'est le potlatch associant deux numaym, et lorsque l'on parle de potlatch inter-tribaux, on ne parle pas de potlatch ayant pour partenaires plusieurs tribus, mais de potlatch inter-numaym tels que chacun des numaym partenaires est soutenu par les autres numaym de sa tribu, l'assistance susceptible de recevoir des biens étants élargie d'autant. C'est un des numaym de la tribu invitante qui distribue des biens à chacun des numaym de la ou des tribus invitées. Ceci doit encore être nuancé. Dans les derniers temps de la vitalité du potlatch sont apparues des formes de cette institution (Mauzé 1985, 1986) qui permettaient à des "nouveaux riches ", à des " hommes forts", de se tailler une place prestigieuse dans la société, mais hors de la norme traditionnelle, à coup de potlatch particulièrement spectaculaires qui, alimentés par le système de prêt à intérêt, brisaient le cadre inter-numaym pour s'inscrire dans un cadre plus large. Vers la fin du xix ${ }^{e}$ siècle (probablement vers 1870 ), la majeure partie des grandes unités cérémonielles se regroupe pour former une sorte de vaste " congrégation rituelle " (Goldman 1975) associant treize tribus. Ces tribus sont hiérarchisées entre elles, huit sont regroupées par paires (ibid. : 33, Boas $1920: 115 ; 1897: 343$; $1966: 81$; Drucker et Heizer $1967: 99-102)$. Cette différenciation de statut entre tribus est non pas fondée sur le mythe, mais liée à des contingences sociohistoriques (contrôle de la traite des fourrures et modalités d'accès à des biens d'origine européenne) ${ }^{16}$. Dans le contexte de rivalité qui oppose les tribus deux à deux, l'ordre hiérarchique peut parfois être bouleversé à la faveur d'un potlatch somptueux, organisé à l'initiative d'un individu de type « nouveau riche ». On a vu que la tribu joue un rôle cérémoniel; à la différence du numaym, elle n'est pas dotée d'un patrimoine symbolique lui conférant son identité, mais elle se manifeste 
cependant comme une unité captatrice de prestige dans les grands potlatch « intertribaux ". Agissant comme un tout englobant pour le compte d'une de ses parties, c'est-à-dire d'un numaym, la tribu, en relation de complémentarité ou d'opposition avec une ou plusieurs tribus, permet aux partenaires du potlatch d'augmenter leur prestige en élargissant leur audience cérémonielle. En fait, les numaym qui ont perdu leur importance sur le plan économique ont tiré moins profit de ce contexte nouveau que, dans la dernière période de l'histoire des Kwagul, quelques individus particulièrement entreprenants.

Le regroupement des numaym au sein de tribus apparaît comme une réponse à la situation faite aux Kwagul avec l'arrivée des négociants européens et ses conséquences économiques et démographiques : le caractère parfois aléatoire des regroupements ne doit pas en masquer la nécessité vitale. Le rôle dévolu à la solidarité militaire dans ces regroupements montre bien que nous avons affaire à un processus d'intégration politique, quels que soient les moyens mis en œuvre pour aboutir à cette intégration et quel qu'ait été l'état d'inachèvement de ce processus à la fin du $\mathrm{XIX}^{\mathrm{e}}$ siècle. Il semble d'ailleurs que la société kwagul, au long du $\mathrm{XIX}^{\mathbf{e}}$ siècle, soit travaillée par des tendances contradictoires : d'une part, une tendance au maintien de l'autonomie du numaym, d'autre part, une tendance à l'intégration tribale. Ce second mouvement semble avoir été lui-même porteur d'un projet politique plus ambitieux encore, dont la constitution de grandes unités cérémonielles pourrait avoir été la première réalisation demeurée sans lendemain, et qui nous apparaît d'ailleurs recéler là encore des tendances contradictoires, comme le montre la coexistence de ces grandes unités et des paires de tribus de rang inégal. On aurait, au cours du $\mathbf{x I X}^{\mathbf{e}}$ siècle, trois idéologies à l'œuvre : une idéologie de la rivalité, dont le référent idéal est la compétition dans le potlatch, transférable, dans des conditions spécifiques, des numaym aux tribus; une idéologie de la hiérarchie, sur laquelle sont fondés les regroupements entre numaym au sein de la tribu, les appariements entre tribus et les agrégations de tribus dans des ensembles cérémoniels, le propre du principe hiérarchique étant à la fois de justifier l'identité de chaque unité, numaym ou tribu, et d'agencer des formes d'associations d'unités de même nature; enfin, une idéologie de la solidarité, plus ou moins intégrative, qui, sur la base d'une mise en commun d'intérêts cérémoniels et/ou militaires, tend à donner forme à des regroupements - de numaym, de tribus qui sont autant de réponses formulées imparfaitement et temporairement aux exigences du moment.

\section{LES SOCIÉTÉS SECRÈTES}

Pendant la saison dite sacrée, période de l'hiver qui correspond aux activités rituelles, l'organisation en numaym est remplacée par une organisation en sociétés secrètes ${ }^{17}$. Introduites chez les Tsimshian vers 1825 et chez les Kwagul méridionaux une dizaine d'années plus tard (Boas 1897 : 664), les sociétés secrètes se sont répandues à partir d'un foyer situé chez les Kwagul septentionaux (Bella Bella et Owikeno); leur présence est beaucoup moins marquée chez les Tlingit, les Nootka et bon nombre de groupes salish (Guédon 1984 : 34-36). 
Les sociétés secrètes regroupent les individus qui ont été initiés à des danses. Chez les Kwagul méridionaux, on distingue principalement quatre sociétés secrètes (Boas 1897 : 394-398), qui correspondent chacune à un " esprit " ou un groupe d'esprits ${ }^{18}$. Placés sous l'emprise des esprits, les initiés reçoivent un nom associé à des privilèges, des droits exclusifs sur des danses et des chants (Boas 1897; Drucker 1940; Guédon 1984). On distingue deux catégories générales d'initiés: les Passereaux et les Phoques. Les Passereaux regroupent l'ensemble des membres « honoraires » des sociétés (initiés « retraités ») qui, une fois qu'ils ont rempli leurs obligations en matière de distribution de biens marquant les différentes étapes de leur initiation, se retirent de la vie cérémonielle active et transmettent leurs positions et leurs titres à des novices. Les Passereaux exercent une fonction rituelle : ils sont chargés d'organiser et de "mettre en scène " les rituels (Boas 1966: 178-179). Statutairement inférieurs aux Phoques, les Passereaux sont subdivisés en plusieurs groupes, auxquels l'appartenance est déterminée en fonction de l'âge et du sexe (Boas $1897: 419)$. Les Phoques sont les initiés " actifs".

Les sociétés secrètes sont hiérarchisées entre elles, la société des Cannibales (hamaća) étant celle dont le statut est le plus élevé. Chaque société regroupe des confréries de danseurs hiérarchisées entre elles (Boas 1897 : 395, 418); chaque confrérie est détentrice du droit à exécuter une danse particulière et de titres associés à ce droit. La hiérarchie des danses correpond dans une certaine mesure, à celle des différents stades de l'initiation au sein d'une même société secrète (Boas 1897 420; Curtis $1915: 159)^{19}$. Pendant la saison cérémonielle, les noms ou titres afférents aux sociétés secrètes remplacent les titres profanes associés au numaym (Boas 1897 : 418).

Modelée sur l'organisation en numaym (Sapir 1967 : 131), l'organisation en sociétés secrètes double et renforce la hiérarchie sociale, en même temps qu'elle transversalise le système des numaym. Pendant la période dite profane, consacrée aux activités économiques (les fêtes se caractérisent alors essentiellement par des distributions de nourriture), la société est divisée en deux grands groupes : les nobles et les non-nobles. Pendant la saison des rituels, c'est la division en initiés et non-initiés qui prévaut. Sur un plan purement formel, ces deux divisions sont homologues l'une de l'autre : les membres (essentiellement des hommes) des sociétés secrètes se recrutent dans la noblesse. L'appartenance aux sociétés secrètes introduit au sein de la noblesse une hiérarchie particulière, puisque les nobles se retrouvent répartis en groupes (sociétés secrètes) et sous-groupes (confréries de danse à l'intérieur d'une société secrète) relevant d'une hiérarchie globale assignant à chacun un rang qui correspond à son grade initiatique. À cet égard, le principe de hiérarchisation qui intervient au niveau du numaym, de la tribu et des sociétés secrètes agit comme une sorte de dynamique centripète, créant le " noyau dur " d'une hiérarchie des hiérarchies.

L'admission des initiés est étroitement liée à la détention de droits héréditaires par les nobles des numaym. Les esprits (esprits cannibale, esprit de la guerre, etc.) qui président aux grandes affiliations initiatiques sont en nombre limité. Ils ne sont pas captés au terme d'une quête individuelle; la relation privilégiée à un esprit fait l'objet d'une transmission par la filiation, l'alliance, ou est acquise dans la guerre (Boas 1897 : 393-394). À la différence des titres associés au numaym, qui restent la 
propriété du groupe et en marquent la pérennité (Boas 1921 : 787, 824; Goldman 1975 : 69), les titres attachés aux sociétés secrètes relèvent de la catégorie des privilèges qui sont notamment obtenus par le mariage (Boas $1921: 840 ; 1935: 93$ ). Les noms "sacrés", transmis par les femmes, confèrent à leurs détenteurs une sorte de pouvoir magico-religieux qui, en retour, joue un rôle essentiel dans le renforcement du statut d'un individu au sein de son numaym.

Les sociétés secrètes tissent un vaste réseau de relations entre nobles co-affiliés. Loin d'être un facteur d'intégration à l'organisation sociale, le système d'appartenance aux sociétés secrètes intervient comme l'élément moteur d'une extrême différenciation entre individus en accentuant les inégalités statutaires, par un effet de superposition et d'accumulation des privilèges, des fonctions et des rôles. On passe de la distinction - propre au numaym - entre un chef et une aristocratie relativement indifférenciée à une gradation de statuts qui, dans la correspondance hiérarchique entre l'ordre des numaym et l'ordre des sociétés secrètes, ou entre l'ordre profane et l'ordre initiatique, surdétermine l'importance des positions les plus élevées. Les personnages de haut rang et notamment les chefs détiennent à la fois le droit à exécuter les danses les plus prestigieuses et à exercer le plus grand nombre de prérogatives rituelles.

Les initiations s'accompagnent obligatoirement de distributions de biens de type potlatch. Á chaque étape de l'initiation d'un enfant, la famille du novice doit donner des biens aux invités témoins de la prise de statut, ce que fait ensuite en son propre nom l'initié devenu adulte. La mise en œuvre d'une initiation exige la disposition d'une grande quantité de richesses, évaluée en fonction du statut de la société d'initiation, de la confrérie de danse au sein de la société et du grade d'initié concerné au sein de cette confrérie. Les distributions s'adressent à une assistance plus large que le numaym ou la tribu; elles visent sinon l'ensemble du moins une grande partie de la communauté des initiés à l'échelle de la société inter-tribale.

L'essor des sociétés secrètes dans la partie centrale de la côte nord-ouest est lié à l'enrichissement des Indiens, du fait de la traite des fourrures, à partir de la fin du $\mathrm{XVIII}^{\mathrm{e}}$ siècle. Le fonctionnement des sociétés secrètes met en évidence la relation nécessaire entre richesse et initiation comme le montre, en creux, la manière dont les sociétés maintiennent l'ordre en leur sein. Les sociétés secrètes protégeaient le secret de leurs activités rituelles. Un initié qui violait " la loi de la danse " ou un non-initié qui avait assisté, même par accident, à une cérémonie qui lui était interdite, pouvait être mis à mort. Mais il arrivait qu'on préférât utiliser d'autres formes de coercition à l'égard de ceux qui trahissaient la loi du secret. Dans certains cas, les sociétés secrètes se transformaient en de véritables associations de malfaiteurs dont l'activité consistait à s'approprier tous les biens des fautifs. Ceuxci étaient soumis au pouvoir d'un ensorceleur et étaient placés sous l'emprise d'une terreur qui se manifestait jusqu'à ce que les richesses du coupable aient été épuisées. Les biens ainsi accumulés par l'entremise d'un chamane maléfique et sous le contrôle de la société secrète étaient redistribués entre les membres de celle-ci (Drucker 1940 : 226).

La détention de positions et des privilèges dans les hiérarchies des numaym et des sociétés secrètes était nécessaire pour consolider un statut. Tout semble indiquer, ne serait-ce que par l'importance qu'ont revêtu les cérémonies d'hiver à partir de la seconde moitié du XIX ${ }^{\mathfrak{e}}$ siècle, que l'appartenance aux sociétés secrètes 
et l'accès à un grade en leur sein ont progressivement pris le pas sur l'accès à un statut au sein du numaym, bien que la détention d'une position au sein d'un numaym ait continué d'être une condition nécessaire pour prétendre à en détenir une dans socitété secrète.

\section{CONCLUSION}

En même temps qu'il y a superposition de deux types d'organisation, ou que l'organisation en sociétés secrètes se rabat sur l'organisation en numaym en lui empruntant son modèle, on perçoit une dynamique à l'œuvre, qui est celle de la consolidation du pouvoir des chefs, qu'il s'agisse des chefs de numaym, détenteurs d'une autorité et d'un pouvoir sur lesquels le consensus est ancien, ou des chefs de tribus, dont le « pouvoir " a été référé à un rôle d'arbitre, de médiateur (Drucker 1983) et finalement de représentant de l'entité tribale. Les sociétés secrètes fournissent vraisemblablement une réponse de type chamanique à la grave crise démographique à laquelle les sociétés de la côte nord-ouest sont confrontées à la fin du XIX ${ }^{e}$ siècle (Kolstee $\left.1988: 135,254\right)$ et dont l'ampleur dépasse sans doute largement le seul retentissement de la diminution de la population sur le fonctionnement de la société. Si cette réponse de type religieux est possible, c'est dans une large mesure grâce à l'afflux de richesses. Les biens d'origine européenne et les revenus monétaires du travail dans le monde euro-canadien qui parviennent dans les tribus sont autant d'éléments de richesse dont la circulation est canalisée par les sociétés secrètes au profit des personnages de très haut rang. Tout indique que l'on a, au cours du xix ${ }^{e}$ siècle, la mise en ouvre d'un processus qui, à partir de l'équivalence ancienne entre pouvoir et prestige (comme richesse symbolique), en vient à identifier ce que l'on pourrait appeler le " pouvoir-prestige " à la richesse réelle, ou à un " pouvoir-richesse », ces deux formes de pouvoir étant unifiées par une même légitimation de caractère religieux ayant son fondement dans l'existence des sociétés secrètes. On aboutit à une nouvelle définition du pouvoir, qui autorise l'apparition, à côté des chefs traditionnels, de personnages qui ne doivent leur rayonnement individuel qu'à leur réussite matérielle. Cette compétition d'un type nouveau entre « chefs " et " nouveaux riches ", qui porte en elle les prémisses de conflits qui seront cruciaux pour la vie politique des communautés des réserves, s'exprime dans un cadre nouveau, qui est celui de la tribu, même s'il y a toujours reversion des enjeux intra-tribaux sur des enjeux intra-numaym. En clair, à la période tardive que nous considérons maintenant, le lieu du politique s'est déplacé du numaym vers la tribu, sur fond de constitution de grands réseaux inter-numaym et inter-tribaux, liée à l'essort des sociétés secrètes.

La question se pose alors de définir dans le langage de l'anthropologie politique ce que sont les deux entités dont nous avons successivement parlé : le numaym et la tribu. L'ensemble des traits caractéristiques que nous avons associés au numaym : territorialité, existence d'un chef héréditaire et d'une aristocratie, hiérarchie de statuts, droit du chef à accumuler des biens prélevés sur les ressources des membres du groupe, capacité d'initiative en matière militaire, évoque une organisation de type " chefferie " (à la fois chiefdom et chieftainship), au sens relativement précis donné aujourd'hui à ce mot, notamment par les anthropologues africanistes ${ }^{20}$. 
Dans la chefferie, les différenciations de statut et de fonction concernent un tissu social relativement homogène, ce qui la rapproche de la société segmentaire, tandis que l'émergence de la figure du chef et la présence d'une aristocratie tire, dans le contexte africain évoqué, vers ce que l'on appellerait un royaume et à propos duquel Davy utilise précisément le terme de « royauté ». En utilisant le terme de royauté, Davy entend à la fois évacuer la problématique de l'état et en même temps mettre en évidence l'émergence ou la possibilité d'émergence d'un principe de souveraineté « sacrée » :

Le chef dont la force physique, dont l'habileté technique, dont les qualités de chasseur et de pêcheur, dont l'aptitude commerciale et la richesse acquise font l'homme qui doit dominer, ne peut dominer véritablement qu'à une condition : c'est qu'il vienne se faire sacrer, pour ainsi dire, dans la confrérie qui seule possède le chrême du pouvoir " (p. 130).

Ce qui caractériserait alors la chefferie kwagul, ce serait une sorte d'équilibre plus ou moins stable qui serait réalisé entre la permanence d'une référence à la parenté, d'une part, l'apparition d'une référence à une unité plus large - qu'il s'agit de définir -, d'autre part. Si nous avons hésité à parler, à propos du numaym de "chefferie " - ou de " micro-chefferie ", sans autre détermination, c'est que le mot apparaît comme un peu trop fort pour désigner une forme d'organisation politique qui n'a jamais concerné qu'un groupe démographiquement très restreint et une territorialité peu homogène. Lorsque nous passons du numaym à la tribu, tout se passe apparemment comme si les conditions nécessaires et suffisantes pour définir cette entité comme une chefferie étaient réunies, au moins en termes de territorialité, en raison de la référence au village d'hiver malgré les réserves que nous avons faites sur la notion d'une territorialité tribale et de taille de la population. Une difficulté de définition surgit cependant. Agrégation hiérarchisée de numaym, la tribu n'est pas une sorte de "numaym des numaym » tel que son chef serait un " chef des chefs ". Nous avons montré quelles étaient les limites de l'intégration tribale, de sorte que la tribu nous est apparue plutôt comme une confédération de numaym que comme une formation englobante et fusionnelle. Doit-on parler, à propos de la tribu, d'une " chefferie sans chef "? La formule, à coup sûr paradoxale, rendrait compte de la permanence d'une autonomie des numaym dans le cadre tribal, mais nullement des caractéristiques du chef de tribu, chef du premier des numaym, dont l'autorité est d'essence rituelle, mais qui, comme garant direct de l'identité tribale et médiateur entre les numaym en temps de paix comme en temps de guerre, se trouve détenteur d'attributions qui relèvent clairement d'un pouvoir de type politique. Sous réserve d'un approfondissement du problème posé ici et d'une investigation étendue aux autres sociétés de la côte nord-ouest, il semble en définitive que l'emploi du mot " chefferie " puisse être utilisé pour désigner ce qui est commun chez les Kwagul, comme forme d'organisation politique, au numaym et à la tribu. Si l'on définit un critère de cohésion interne s'exprimant dans l'unité du groupe et l'étroite articulation du groupe à son chef, et un critère de cohésion externe rendant compte de la capacité du groupe à faire la guerre, numaym et tribu, en tant que « chefferies ", semblent marqués d'incomplétude, mais dans des registres opposés. Tandis que le numaym relève positivement du premier critère, il est dans un rapport négatif au second - sa faible assise territoriale et démographique le rend 
vulnérable -; la tribu offre l'exemple d'une situation exactement inverse : faiblement intégrative, elle dispose cependant des moyens de la guerre défensive et offensive. À la fin du $\mathrm{xIx}^{\mathrm{e}}$ siècle, les forces d'intrégration tribale semblent l'emporter sur les forces qui, si l'on peut dire, tirent la société vers les numaym, dans un contexte général d'individualisation du pouvoir et d'émergence d'hommes forts à l'échelle de la tribu. Dans le même temps, un second processus peut être identifié qui est celui de la montée en puissance des sociétés secrètes. On peut se demander alors, si entre la base sociale des numaym et les nouveaux pouvoirs individualisés dont celui de chef de tribu, les sociétés secrètes n'ont pas joué une rôle de corps intermédiaire à vocation rituelle. Les sociétés secrètes auraient rempli une double fonction de maintien de l'identité symbolique des numaym et de légitimation rituelle de l'existence de la tribu*.

Article reçu en mars 1989, accepté pour publication en juin 1989.

\section{NOTES}

1. Les " totems" sont en fait des blasons, généralement des figurations animales, qui sont représentées sur les mâts totems, les frontons de maison, les coffres cérémoniels, etc. Ce sont des objets appropriés individuellement et susceptibles d'être échangés; plus que la marque d'une appartenance à un groupe, ils expriment certains traits afférents au statut social.

2. Cf. Mauzé 1986; Schulte-Tenckhoff 1986.

3. Dans la Foi jurée (1922), Davy note : « Le potlatch est non le substitut, mais le complément, et, pouvons-nous ajouter, le correctif de l'hérédité. Il est dans la société la promotion au choix à côté de l'ancienneté. 11 pousse les hommes nouveaux que la richesse favorise. C'est lui que nous retrouvons, au même titre, comme facteur de la hiérarchie dans les sociétés secrètes où nous découvrirons, en effet, une pépinière de l'individualisme nouveau de la richesse en même temps qu'un lieu de consécration de l'individualisme ancien de la naissance. Envisagé de la sorte, le potlatch est donc véritablement le facteur social d'un d'ordre nouveau " (234-235).

4. Dans son dictionnaire du kwakwala (1948, ms : 240), Boas donne du numaym la définition suivante : « family group supposed to be descendants of one ancestor, but assignable in paternal and maternal line, also including adopted members ".

5. Le terme bekwa'x signifie « homme de bas rang "; beg'wElede : « homme de la maison " (celui qui appartient au chef) (Boas 1948 : 113).

6. Les esclaves sont des captifs de guerre, généralement issus d'un groupe ethnique voisin. Plusieurs études - notamment Donald (1984, 1985), Mitchell $(1984,1985)$, Mitchell et Donald (1985) - mettent en relief l'importance du rôle économique des esclaves dans les sociétés de la côte nord-ouest. Cependant, le statut des esclaves au sein de ces populations demanderait à être défini avec précision. Chez les Nootka, les chefs font des esclaves de loyaux suivants. Ce ne semble pas être le cas chez les Kwagul. Le retour d'un prisonnier de guerre noble dans sa communauté d'origine pouvait être obtenu contre rançon (umista). Dans les années 1840 , on évalue le nombre d'esclaves chez les Kwagul à environ $3,5 \%$ de la population d'un groupe.

7. La hiérarchie des statuts opère à l'intérieur même de la parenté. Ceux dont le rang de naissance était défavorable en vertu de l'application du principe de primogéniture devenaient les gens de la maison du chef (Boas $1966: 53$ ). Le chef de guerre du numaym était le plus jeune frère du chef de numaym, ce qui lui permettait, au lieu d'être relégué au rang d'homme du commun, de jouir d'un statut particulier (Boas 1966 : 106).

8. Haggerty et Inglis (1986) remettent en cause la thèse des déplacements saisonniers pour les Nuchanulth (Nootka) de la côte ouest de l'île de Vancouver. En s'appuyant sur des données archéologiques et ethno-historiques, ces deux auteurs avancent l'hypothèse selon laquelle, avant le contact, les activités de 
cueillette, de pêche et de chasse étaient conduites à partir d'un village permanent, les sites de production se trouvant tout près du village. Les produits alimentaires manquants étaient obtenus par troc et lors des fêtes (pour une étude récente de la territorialité nootka, cf. Golla 1987). L'application d'un tel modèle aux Kwagul vaut la peine d'être pris en considération.

9. Un informateur de Drucker fait les remarques suivantes : "Since about 1900 our people has changed. Before each namima has leader, the people had to give him whatever they could to him (just like government get tax now).... In recent times is all individual chief would raise money by himself, but that is just new way " (sic) (Drucker 1953). À partir de la deuxième moitié du XIXe siècle, les potlatch sont financés par un système de prêts et de remboursements à intérêt. C'est ce que les Kwagul appellent le "business potlatch ». Au moment de l'organisation d'un potlatch, le chef invitant oblige ses débiteurs à rembourser leurs dettes au double.

10. Dans le premier recensement (1835) des populations indiennes de la région qui allait devenir la Colombie britannique, Tolmie (traitant de fourrure de la Compagnie de la Baie d'Hudson) établit une liste de groupes kwagul qui sont non pas des numaym mais ce qu'il est convenu d'appeler des tribus; à propos des journaux de Fort Langley (1827-1830), cf. Mauzé (1985: 335-337).

11. Il n'existe pas en kwakawala (langue des Kwagul) un terme spécifique qui désignerait l'entité que nous appelons " tribu ». Boas traduit le terme kwakwala g. $0^{\prime} k w E l o t$ par " communauté villageoise " (1890: 56), ou « habitants des maisons " (1966: 37). Ce terme s'oppose à un autre terme le'lkolale, qui signifie : "Ceux qui ont des feux qui se déplacent sur l'eau » (Boas, ibid.), et qui désigne en fait le village voisin. Codere (1961 : 442) utilise le terme de village, Donald et Mitchell (1975: 326), celui de groupe local; cf. aussi Boas (1920:111-12). Les Kwagul de Fort Rupert et les Lekwiltoq sont subdivisés en sous-tribus, comprenant chacune plusieurs numaym (Mauzé 1985).

12. Peu d'attention a été portée à l'étude de la guerre dans les société de la côte nord-ouest, du moins jusqu'à ces dernières années marquées par les travaux de Donald et Mitchell $(1975,1984,1985)$ et de Ferguson $(1979,1984)$. Ces auteurs ont montré qu'avant la période dite historique, la guerre était un phénomène constitutif de la vie de ces sociétés et était directement liée à l'acquisition de nouveaux territoires ou au contrôle de territoires abondants en ressources halieutiques (voir aussi Swadesh et Sapir, 1948). Sur la base de données archéologiques, MacDonald (1979:11) émet l'hypothèse selon laquelle les populations de la côte nord-ouest ont vécu dans un état de guerre endémique depuis le début du premier millénaire avant J.-C. Après le contact (fin XviII ${ }^{e}$ siècle) et le développement de la traite des fourrures, les guerres s'intensifient en raison de l'introduction de nouvelles armes mais surtout d'une importance nouvelle des enjeux économiques : contrôle du commerce par les groupes situés près des routes et des postes de traites, pillages, expéditions à longue distance en vue de la capture d'esclaves par les groupes situés loin des circuits de traite, les captifs étant destinés à être échangés contre des biens d'origine européenne.

13. L'ordre chronologique d'affiliation des numaym à une tribu a valeur de principe de hiérarchisation. Boas cite l'exemple de deux numaym qui, après s'être détachés de leur tribu d'origine pour s'agréger à une autre tribu, sont relégués aux deux derniers rangs dans la tribu d'accueil $(1897: 330 ; 1921: 801)$.

14. Dans les distributions traditionnelles, l'ordre selon lequel les biens étaient distribués suivait l'ordre hiérarchique des statuts des individus au sein des numaym et des numaym au sein des tribus. Dans le contexte du développement des distributions intertribales, de l'augmentation du volume des biens distribués, de l'apparition des "nouveaux riches " qui s'immiscent dans les potlatch, est crée l" " ordre " des Aigles, qui est censé regrouper " ceux qui sont servis en premier ». Cet ordre institue une hiérarchie supplémentaire, mais qui n'intervient que dans les distributions. Les positions au sein de l'ordre des Aigles sont hiérarchisées entre elles (Mauzé $1986: 26-27 ; 42$ ).

15. Les guerres de conquête et les raids à longue distance nécessitaient la mobilisaton d'un nombre important de guerriers et la mise en place d'un commandement unique. Sur le terrain, c'est le chef de la guerre qui mène les opérations avec le concours de quelques guerriers professionnels. Seuls les esclaves étaient contraints de partir au combat ; les guerriers de statut libre sont des volontaires (Boas $1966: 107$; Curtis $1915: 103)$. On compte environ un guerrier professionnel pour huit à dix guerriers non spécialisés, volontaires ou non : ceux-ci sont rameurs, guetteurs, pilleurs, etc. (Curtis 1915: 100). Lors des déplacements sur mer, les équipages sont constitués par des membres d'un même numaym. Dans les guerres tardives conduites à l'échelle tribale ou intertribale, les pertes tendaient à se répartir à peu près également entre les numaym qui participaient à l'action des groupes constitutifs de la tribu.

16. L'exemple des Kwagul de Fort Rupert qui bénéficient du statut le plus élevé dans le système hiérarchique des tribus est éloquent. En venant s'installer à Fort Rupert (poste de traite de la Compagnie de la Baie d'Hudson), les sous-tribus qui forment la tribu des Kwagul de Fort Rupert s'enrichirent 
considérablement en contrôlant le commerce des foursures. Déjà, avant le développement de la traite des fourrures, l'ensemble des numaym qui composent les sous-tribus détenaient, dans cette région, des sites de pêche où le saumon était particulièrement abondant (Donald et Mitchell 1975).

17. Cf. aussi la définition de Drucker $(1940: 201)$. Une étude plus approfondie sur la nature des sociétés secrètes reste à faire; elle montrerait sans doute que l'emploi de l'expression "société secrète " n'est pas tout à fait approprié pour désigner ces "sociétés de danse " kwagul.

18. Les esprits qui président aux sociétés secrètes sont : Bax̌bakwalanuksiwe (esprit cannibale), Winalagilis (esprit de la guerre), Mádem (oiseau qui octroie la possiblité de voler) et les esprits-fantômes (Boas 1897 : 394-395).

19. Ce que nous avons désigné sous l'appellation « confrérie de danseurs 》 est nommé " société » par Boas $(1897$; 1966), « ordre " par Curtis (1915) et Drucker (1940). La société secrète des Cannibales, placée sous l'influence de Bax́bakwalanuksiwe, comprend plusieurs confréries de danseurs, entre autres : les hamaća (les Cannibales), les nutmat (les Possédés), les Ours-Grizzlis, etc.

20. Pour la définition des chefferies, voir notamment : Balandier (1967), Service (1962), Sahlins (1967, 1968), Fried (1967), Lewellen (1983), Spencer (1983) et Terray (1985). Le cas de la côte nord-ouest est traité particulièrement par Service $(1962 ; 1978)$ et Lewellen, ainsi que Toffelson (1986).

\section{BIBLIOGRAPHIE}

Balandier, G., 1967. - Anthropologie politique. Paris, P.U.F.

BOAS, F., 1890. - « First General Report on the Indians of British Columbia ». Report of the 59th Meeting of the British Association for the Advancement of Science for the year 1889 , London

-, 1897. - «The Social Organization and the Secret Societies of the Kwakiutl Indians ». Report of the U.S. National Museum for 1895, 331-738, Washington.

-, 1920. - « The Social Organization of the Kwakiutl ». American Anthropologist, New Series, 22/2, 111-126.

-, 1921. - «Ethnology of the Kwakiutl », Bureau of American Ethnology, Thirty-Fifth Annual Report, parts 1 \& 2, Smithsonian Institution, Washington.

-, 1935. Kwakiutl Culture as Reflected in Mythology. New York, The American Folk-Lore Society, G. E. Stechert ("Memoirs of the American Folklore Society " XXVIII).

-, 1948. - Kwakiutl Dictionary, ed by H. Boas Yampolski, American Philosophical Society, ms $\mathrm{n}^{\circ} 30$, w 1 a. 21.

-, 1966. - Kwakiutl Ethnography, edited by H. Codere, Chicago, Chicago University Press.

BoYD, R. T., 1985. - The Introduction of Infectious Diseases among the Indians of the Pacific Northwest 1774-1874. Unpublished Ph. D. Thesis, Seattle, University of Washington.

Carroll, M. P., 1980. - « Lévi-Strauss on Art : A Reconsideration ", Anthropologica, XXII/2, 203-214.

Codere, H., 1950. — « Fighting with Property ». Monographs of the American Ethnological Society, $\mathrm{n}^{\circ} 18$. Seattle, University of Washington Press.

-, 1961. - « Kwakiutl ». Perspectives in American Indian Culture Change, E. Spicer (ed.), Chicago, University of Chicago Press, 431-516.

CUrtis, E. S., 1915. - The Kwakiutl. The North American Indian. Vol. 10. Nordwood, Mass, The Plimpton Press.

Davy, G., 1922. - La Foi Jurée. Paris, Félix Alcan. 
Dawson, G. M., 1887. - « Notes and Observations on the Kwakiool People of the Northern Part of Vancouver Island and Adjacent Coasts, Made during the Summer 1885 (...) ". Proceedings and Transactions of the Royal Society of Canada for the year 1887, 5/2, 63-98.

Denman, R. \& C. URIBE (eds.), 1983. - Chiefdoms in the Americas. New York, University Press of America.

Donald, L., 1983. - «Was Nuu-chah-nulth-aht (Nootka) Society Based on Slave Labor? », in Tooker et Fried (eds.), 108-119.

-, 1984. - "The Slave Trade on the Northwest Coast of North America ». Research in Economic Anthropology, 6, 121-158.

-, 1985. - « Captives or Slaves? A Comparison of Northeastern and Northwestern North America by Means of Captivity Narratives ", Culture, V/2, 17-23.

Donald, L. \& D. H. Mitchell, 1975. - « Some Correlates of Local Group Rank among the Southern Kwakiutl ». Ethnology, 14/4, 325-346.

Drucker, P., 1939. - « Rank, Wealth, and Kinship in Northwest Coast Society ». American Anthropologist, N.S., 41, 55-65.

-, 1940. - « Kwakiutl Dancing Societies». University of California Anthropological Records (6), Berkeley, University of California Press, 201-230.

-, 1953. - Unpublished Field Notebooks from B.C. Box 1 (2), vol. 1, B.C. Provincial Archives, Victoria.

—, 1983. - « Ecology and Political Organization on the Northwest Coast of America ». In Tooker (ed.), 86-96.

Drucker, P. \& R. F. HeIzer, 1967. - To Make My Name Good. Los Angeles, University of California Press.

FERguson, B., 1983. - «Warfare and Redistributive Exchange on the Northwest Coast ». In Tooker (ed.) : 133-147.

-, 1984. - «A Reeamination of the Causes of Northwest Coast Warfare ». In Warfare, Culture and Environment, R. B. Ferguson (ed.), New York, Academic Press Inc., 267328.

FrIed, M., 1967. - The Evolution of Political Society. New York, Random House.

Goldman, I., 1975. - The Mouth of Heaven. New York, John Wiley \& Sons.

Golla, S., 1987. - He Has A Name: History and Social Structure among the Indians of Western Vancouver Island. Unpublished Ph. D. Dissertation, New York, Columbia University.

Gú́bon, M. F., 1984. - « La Formation des sociétés secrètes amérindiennes de la côte nord-ouest ». Recherches amérindiennes au Québec, XIV/2, 35-45.

HALPIN M., 1973. - The Tsimshian Crest System : A study based on Museum Specimens and the Marius Barbeau and William Beynon Filed Notes. Unpublished Ph. D. Dissertation, Vancouver, University of British Columbia.

Inglis, R. \& J. C. Haggarrty, 1986. - « Historic Resources Site Survey and Assessment, Pacific Rim National Park ». Ms, Parks Canada, Calgary.

Kolstee, A., 1988. - To Impersonate the Supernatural. Music and Ceremony of the Bella Bella (Heiltsuq) Indians of British Columbia. Unpublished Ph. D. Thesis, UrbanaChampaigne, University of Illinois.

Laguna (de) F., 1983. - « Aboriginal Tlingit Sociopolitical Organization ». In Tooker (ed.), 71-85. 
LANDO, P., 1988. - The Socio-History of the Units of Kwakiutl Property Tenure. Thesis submitted in partial fulfillment of the requirements for the Degree of Master of Arts, Vancouver, University of British Columbia.

LEVI-STrauss, C., 1979. - « L'Organisation sociale des Kwakiutl ». In La Voie des masques, édition revue, augmentée et rallongée de trois excursions. Paris, Plon.

-, 1984. - Paroles données. Paris, Plon.

Lewellen, T. C., 1983. - Political Anthropology. South Haddlet, Mass., Bergin \& Garvey Publishers Inc.

LowIE, R. H., 1967. - « Some Aspects of Political Organization among the American Aborigines ". In Comparative Political Systems, R. Cohen and J. Middleton (eds.), Austin, University of Texas Press, 63-87.

MauzÉ, M., 1985. - Enjeux et jeux du prestige. Des Kwagul méridionaux aux Lekwiltoq (côte nord-ouest du Pacifique). Thèse de $3^{\mathbf{e}}$ cycle. Paris, EHESS.

-, 1986. - «Boas, les Kwagul et le potlatch ». L'Homme, XXVI/100, 21-64.

-, 1988. - « The Concept of the person among the Kwagul Indians », communication présentée dans le cadre du symposium organisé par S. Golla et P. Whiteley "Persons and selves in Pueblo and Northwest Coast Societies. Marcel Mauss Revisited ", American Anthropological Association Annual Meeting, Phoenix, novembre 1988.

MacDonald, G., 1979. - Kitwanga Fort National Historical Site, Skeena River, British Columbia. A Historical Research and Analysis of Structural Remains. Ottawa, National Museum.

-, 1980. - The Epic of Nekt. The Archeology of Metaphor. Banquet address to the 13th Annual Meeting of the Canadian Archeological Association, Saskatoon, Saskatchewan.

MacLeOD, W. C., 1928. - "Economic Aspects in Indigenous American Slavery ». American Anthropologist, 30, 622-650.

Mitchell, D., 1983. - « Seasonal Settlements, Village Aggregations, and Political Autonomy on the Central Northwest Coast ". In Tooker (ed.), 97-107.

-, 1984. - «Predatory Warfare, Social status, and the North Pacific Slave Trade ». Ethnology, XXIII/1, 39-48.

--, 1985. - «A Demographic Profile of Northwest Coast Slavery ». In Status, Structure and Stratification : Current Archeological Reconstructions, M. Thompson, M. T. Garcia, F. J. Kense (eds.), Proceedings of the Sixteenth Annual Conference, the University of Calgary, Archeological Association, 227-236.

Mrtchell, D. \& L. Donald, 1985. - « Some Economic Aspects of Tlingit, Haida, and Tsimshian Slavery ». Research in Economic Anthropology, 7, 19-35.

MORET, A. \& G. Davy, 1922. - Des Clans aux empires. Paris, La Renaissance du livre, " coll. l'Évolution de l'humanité ».

SAhlins, M., 1963. - «Poor Man, Rich Man, Big Man, Chief : Political Types in Melanesia and Polynesia ». Comparative Studies in Society and History, 5, 285-303.

-, 1967. - «The Segmentary Lineage Organization of Predatory Expansion ". In Comparative Political Systems, R. Cohen \& J. Middleton (eds.), Austin, University of Texas Press.

-, 1968. - Tribesmen. Englewood Cliffs, Prentice-Hall, Inc.

SAPIR, E., 1979. - « L'Organisation sociale des tribus de la côte ouest ». In Anthropologie 2. Culture, E. Sapir, Paris, Les Éditions de minuit, présentation de Ch. Baudelot. 
SAPIR, E. \& SWADESH, 1948. - « Motivations in Nootka Warfare ». Southwestern Journal of Anthropology, 4, 76-93.

Schulte-Tenckoff, I., 1986. - Potlatch : conquête ou invention. Lausanne, Editions d'en bas, "Coll. Forum anthropologique ".

Service, E., 1962. - Primitive Social Organization. New York, Random House.

-, 1978. - Profiles in Ethnology (« The Nootka of British Columbia »). Santa Barbara, University of California (3rd. edition).

SPENCER, C., 1983. - " Rethinking the Chiefdom ». In Denman \& Uribe (eds.) :

TAYlor, H. \& W. Duff, 1956. - «A Post-Contact Southward Movement of the Kwakiutl ». Research Studies of the State College of Washington, 24/1, 55-66.

Terray, E., 1985. - « Sociétés segmentaires, chefferies, états : acquis et problèmes ». Revue canadienne des études africaines, 19/7, 106-115.

Testart, A., 1982. - Les Chasseurs-cueilleurs ou l'origine des inégalités. Paris, Société d'ethnographie.

-, 1985. - Le Communisme Primitif 1. Économie et idéologie. Paris, Éditions de la Maison des Sciences de l'Homme.

Toffelson, K., 1986. - «The Snoqualmie : A Puget Sound Chiefom ». Ethnology, 28/2, 121-136.

TOOKer, E. \& M. FrIed, 1983. - The Development of Political Organization in Native North America. 1979. Proceedings of the American Ethnological Society, Washington, American Ethnological Society.

WIKE, J., 1967. — « The Role of the Dead in Northwest Coast Culture ». In Indians tribes of Aboriginal America, S. Tax (ed.), New York, 97-103. 